\title{
ENTRE EUROPA Y AMÉRICA LATINA: MERCEDES RODRIGO, PSICOPEDAGOGA PIONERA ${ }^{1}$
}

\author{
Ana Guil Bozal ${ }^{2}$ \\ Universidad de Sevilla - España \\ Grupo de investigación HISULA \\ anaguil@us.es \\ Sara Vera Gil ${ }^{3}$ \\ Universidad de Sevilla - España \\ sarvergil@alum.us.es
}

Recepción: 28/08/2010

Evaluación: 09/05/2011

Aceptación: 22/10/2011

Artículo de Reflexión

\section{RESUMEN}

Partiendo de la hipótesis de la amplia influencia que los trabajos de Mercedes Rodrigo Bellido han tenido en la Psicología y la Pedagogía, tanto española como colombiana, nos dispusimos a llevar a cabo el presente trabajo de investigación. Nuestro objetivo general fue visibilizar la figura de esta

${ }_{1}$ El artículo que presentamos forma parte del trabajo que desde hace años viene desarrollando el Grupo de Investigación HUM-219: Género y Sociedad del Conocimiento del que la primera autora es responsable (integrado en el Plan Andaluz de Investigación, Desarrollo e Innovación de la Junta de Andalucía, España). Grupo de investigación que tiene como uno de sus objetivos prioritarios, visibilizar la labor que las mujeres han desarrollado a lo largo de la Historia en distintas disciplinas tales como, en esta ocasión, la Psicología y la Pedagogía. Para esta investigación se contó con la financiación general del Grupo y se llevó a cabo desde la Universidad de Sevilla, en concreto desde el Departamento de Psicología Social de la Facultad de Psicología. El proceso de búsqueda bibliográfica se desarrolló a través de bases de datos especializadas como Dialnet, Psicodoc, PsycINFO, ERIC, PsyARTICLES, Psyc BOOKs.

2 Doctora en Psicología Social de la Universidad de Sevilla - España, integrante de los grupos de investigación Historia y Prospectiva de la Universidad Latinoamericana HISULA categoría A1 COLCIENCIAS de la Universidad Pedagógica y Tecnológica de Colombia y del grupo Género y Sociedad del Conocimiento, es autora de 10 libros, 30 capítulos de libro y varios artículos en revistas indexadas.

3 Estudiante de Psicología. Universidad de Sevilla-España, grupo de investigación Género y Sociedad del Conocimiento Línea de investigación: Género, mujeres y poder. 
autora a través de la revisión de su obra, así como de los escritos que sobre ella han dejado otras autoras, como Fania Herrero, quien elaboró su tesis doctoral en torno a esta psicopedagoga semi-oculta en el pasado.

Tras la revisión de los textos seleccionados encontramos que la figura de Mercedes Rodrigo Bellido fue especialmente importante para la Psicología colombiana, siendo su mayor contribución la creación del primer programa de formación en Psicología, que pudo extender por toda América Latina.

Así mismo, fue artífice de otras muchas importantes contribuciones relacionadas especialmente con la creación de puentes entre la psicopedagogía europea y la española, o con la toma de conciencia de que la Psicología tenía que tener su propio campo de trabajo, ya que sus contenidos venían siendo abordados desde diversas especialidades tales como la Medicina o la Teología. En España, tuvo además un importante papel en los inicios de la Psicología Aplicada. Todas sus estas importantes aportaciones de Mercedes Rodrigo Bellido contrastan con la escasa atención que hasta ahora esta eminente psicopedagoga madrileña ha tenido en nuestro campo de conocimiento.

Palabras clave: Revista Historia de la Educación Latinoamericana, Europa y América Latina, Mercedes Rodrigo.

\title{
BETWEEN EUROPE AND LATIN AMERICA: RODRIGO MERCEDES, PSYCHOLOGIST PIONEER
}

\author{
Ana Guil Bozal \\ Universidad de Sevilla - España \\ Research group HISULA \\ anaguil@us.es \\ Sara Vera Gil \\ Universidad de Sevilla - España \\ sarvergil@alum.us.es
}

\begin{abstract}
Assuming the influence that the research of Mercedes Rodrigo Bellido has had on the psychology and Spanish and Colombian pedagogy, we are going to explain this research. Our overall objective was to make visible the figure of the author through the revision of hes work, as well as the
\end{abstract}


writings of other authors have left it as Fania Smith, who prepared hes doctoral thesis around this semi-occult psychologist.

Following a review of selected texts we find that the figure of Mercedes Rodrigo Bellido was especially important for the Colombian Psychology, and her main contribution was the creation of the first training program in psychology, which could extend throughout Latin America. She also was the author of many important contributions especially related to building bridges between the European and Spanish psychology, or the realization that psychology had to have its own field of work, since their contents were being approached from various fields such as medicine or theology. In Spain, also had an important role in the beginnings of Applied Psychology.

All these important contributions from Mercedes Rodrigo Bellido opposite with the limited attention so far this eminent psychologist Madrid has had on our field of knowledge.

Key words: Journal of Latin American Education History, Europa y América Latina, Mercedes Rodrigo.

\title{
ENTRE EUROPA E AMÉRICA LATINA: MERCEDES RODRIGO, PSICOPEDAGOGA PIONEIRA
}

\author{
Ana Guil Bozal \\ Universidad de Sevilla - España \\ Grupo de pesquisa HISULA \\ anaguil@us.es \\ Sara Vera Gil \\ Universidad de Sevilla - España \\ sarvergil@alum.us.es
}

\section{RESUMO}

Partindo da hipótese da ampla influência que os trabalhos de Mercedes Rodrigo Bellido tiveram na Psicologia e na Pedagogia, tanto espanhola quanto colombiana, nos dispusemos a levar a cabo o presente trabalho de pesquisa. Nosso objetivo geral foi dar visibilidade à figura desta autora através da revisão de sua obra, assim como dos escritos que sobre ela produziram outras autoras, como Fania Herrero, quem elaborou tese 
doutoral sobre esta psicopedagoga semi-oculta no passado. Para alcançar este objetivo, a metodologia utilizada foi o levantamento bibliográfico em bases de dados especializadas, tais como Psicodoc, PsycINFo, ERIC, PsyARTICLES, PsycBOOKs e TESEO.

A partir da revisão dos textos selecionados das bases de dados comentadas, concluímos que a figura de Mercedes Rodrigo Bellido foi especialmente importante para a Psicologia colombiana, sendo sua maior contribuição a criação do primeiro programa de formação em Psicologia, estendido por toda América Latina. Assim mesmo, foi artífice de outras muitas e importantes contribuições, relacionadas especialmente com a criação de pontes entre a psciopedagogia européia e a espanhola, ou com a tomada de consciência de que a Psicologia deveria possuir seu próprio campo de trabalho, já que seus conteúdos vinham sendo abordados a partir de diversas especialidades tais como a Medicina ou a Teologia. Na Espanha, teve, ademais, um importante papel nos primórdios da Psicologia Aplicada.

Todas estas importantes contribuições de Mercedes Rodrigues Bellido contrastam com a escassa atenção que até agora recebeu esta eminente psicopedagoga madrilenha em nosso campo de conhecimento.

Palavras- chave: Revista História da Educação Latino-americana, Europa y América Latina, Mercedes Rodrigo.

\section{INTRODUCCIÓN}

La historia de las mujeres sigue siendo a día de hoy una asignatura pendiente (Boix, 2005). Las contribuciones que hemos hecho en todas las disciplinas y campos de conocimiento han quedado siempre ocultas tras los hombres, ostentado ellos todos los méritos, premios y reconocimientos. Como nos dice Nuria Solana (Solana, 2002, citada en Boix, 2005) la recuperación de espacios de libertad femeninos pasa por la reconstrucción de la genealogía de las mujeres en las distintas áreas del saber, pues es de justicia que tras siglos de invisibilidad se reconozcan de una vez los nombres de aquellas que contribuyeron al avance de la humanidad. El homenaje y la recuperación de la vida y obra de estas mujeres sobresalientes son, por lo tanto, merecidos y necesarios. No se pretende con ello, sin embargo, construir una historia paralela sino rellenar los vacíos que han quedado en el esfuerzo del patriarcado por ocultar y apartar a las mujeres de la ciencia.

Bajo este marco, el propósito general de la presente revisión ha sido profundizar en la figura de Mercedes Rodrigo Bellido (Madrid 1891Puerto Rico 1982) psicopedagoga española pionera, formada en Europa, 
que desarrolló la mayoría de su trayectoria profesional en América Latina, representando como nadie la conexión entre la Escuela Nueva y la Psicología Aplicada. Tras resumir su formación, analizaremos los inicios de su desarrollo profesional en España, para centrarnos después en su labor profesional en Colombia y Puerto Rico, países a los que se exilió tras la guerra civil española. Es de destacar especialmente el importante trabajo que realizó en Colombia en donde -pese a vivir sólo 11 años- entre otras muchas actividades, fue la responsable de la creación del primer programa profesional de Psicología de su Universidad Nacional.

Para acercarnos a la figura de esta importante psicopedagoga hemos realizado una extensa revisión bibliográfica, tanto de su obra como de la de quienes han trabajado sobre ella, especialmente desde Colombia Rubén Ardila y desde España Fania Herrero, que precisamente realizó su Tesis Doctoral sobre nuestra pionera, analizando minuciosamente toda la documentación pública existente en archivos y registros.

Realmente la intención final de este trabajo ha sido mantener el interés -activado hace poco más de una década- por este importante personaje de la Historia de la Psicopedagogía europea y americana que, como muchas otras de sus colegas, se encuentra semi-oculta en el pasado. Si con ello conseguimos avivar la curiosidad por su vida y por su obra, habremos cumplido nuestra misión. Rescatar a ésta y a tantas otras mujeres que contribuyeron a forjar nuestras actuales instituciones científicas y educativas, nos parece un necesario acto de merecido reconocimiento a la vez que de infinito agradecimiento.

\section{Orígenes y formación inicial}

Mercedes Rodrigo Bellido nació en Madrid el 12 de mayo de 1891, hija de Pantaleón Rodrigo y Falces y de María Bellido Díaz, ambos naturales de la provincia de Navarra. Su padre era profesor de música, afición que heredó su hermana María, 3 años mayor que ella, que con los años llegaría a ser no sólo profesora, sino también reconocida compositora y pianista. Mientras que ella se inclinaría más por la docencia.

Su infancia y juventud transcurrió en Madrid. Como era frecuente entre las chicas que en sus tiempos querían tener formación universitaria, comenzó estudiando en la Escuela Normal, donde terminó sus estudios en 1910, precisamente el año en que -ante la avalancha de peticiones- se dio el respaldo legal a la entrada oficial de las mujeres en las universidades españolas. En Julio de 1911 obtuvo su título de Maestra Superior, después de aprobar los 3 ejercicios de que constaba la reválida del grado superior. 
Durante algunos años más continúo su formación en diversos centros de educación especial en Madrid, con extraordinarias calificaciones, visitando en 1913 algunas instituciones educativas europeas para personas deficientes, sordomudas y ciegas en Alemania, Francia y Bélgica, viaje que hubo de interrumpir ante la inminente primera guerra europea. También durante esta época, asistió regularmente -entre 1915 y 1917- al Curso Permanente de Dibujo de la Sección de Maestros.

En 1919 es nombrada profesora interina de la sección de Labores y Economía Doméstica del Colegio de Ciegos de Madrid, cargo al que renuncia cuando le conceden, el 15 de septiembre de1920, una Pensión de la Junta para Ampliación de Estudios de la Institución Libre de Enseñanza con la que financiar -a lo largo de 18 meses- sus estudios Psicopedagógicos en Ginebra.

\section{Formación europea}

El 16 de octubre de 1920 Mercedes Rodrigo Bellido viaja a Suiza y se matricula oficialmente en $L^{\prime} E$ cole des Sciences de L'Education del Instituto Rousseau, dirigido por E. Claparéde, que ese mismo año fundaría la Asociación Internacional de Psicología Aplicada y que sería -con toda certeza- la figura europea que más influiría en ella. Y como tenía previsto, inicia sus estudios en la Universidad de Ginebra, en la que cursa las siguientes materias para la obtención de su licenciatura:

- Psicología Experimental. Prof. Edouard Claparéde.

- Técnica Psicológica. Prof. Jean Piaget.

- Orientación Profesional. Prof. Heinis.

- Patología y Educación de Anormales. Prof. Naville y Alice Descoeudres.

- Psicología y Pedagogía de los sentimientos morales. Prof. Pierre Bovet.

- Sugestión y fuerza moral. Prof. Ch. Badouin.

- Psicoanálisis educativo. Prof. Sabina Spielrein.

- Pedagogía Experimental. Prof. Marjorie Duvillard.

Cabe señalar cómo, ya a principios del siglo XX, habían mujeres psicólogas no sólo ejerciendo la profesión sino también impartiendo clases en la Universidad, pese a que en la inmensa mayoría de libros de Historia de la Psicología se citen exclusivamente a personajes masculinos. Durante todo el tiempo que duró su beca, Mercedes colaboró con Claparéde en el Laboratorio de Psicología de la Universidad, hizo prácticas de educación especial en la Maison des Petits, y prácticas pedagógicas en los centros anejos al Instituto Rousseau, trabajando así mismo en el Gabinete de Orientación Profesional. 
Todas las referencias indican que tanto ella como el resto de becadas y becados, eran excelentes estudiantes que participaban activamente en la comunidad universitaria pues, a la vez que recibían formación, colaboraban en investigaciones, impartiendo algunas clases y ayudando, o redactando ellos mismos, múltiples publicaciones.

Su inquieta personalidad científica, a la que nada parecía ajeno, la llevó durante el periodo de su formación de la pedagogía práctica a la psicotecnia y la psicología experimental, abarcando la psicología infantil, la psicología de anormales y la orientación profesional.

Entre sus compañeros destaca la figura de otra mujer, Mme. Loosli-Usteri, que poco después sería copresidenta -junto con Pierre Bovet- de la Sección Social de la Unión Internacional de Socorro a los Niños, que posteriormente estaría representada a su vez en América Latina por quien había sido precisamente su profesora de Pedagogía Experimental, Marjorie Duvillard.

Poco antes de su regreso a España publicó una investigación sobre el pensamiento infantil acerca de la guerra de Marruecos, trabajo que realizó con su compañero de beca Pedro Roselló, en el que mostraron su complacencia con que, la mayoría de los 819 chicos y chicas de entre 8 y 15 años que encuestaron, fueran poco partidarios de las guerras, algunos incluso pacifistas, especialmente los de clases bajas ${ }^{5}$. Es interesante observar cómo en aquellos años, se anticiparon ya a un tema de absoluta actualidad en nuestros días: la necesidad de Educación para la Paz.

Finalmente -en 1923- se diplomó en Psicología en la Universidad de Ginebra, terminando su pensión en el extranjero con una visita durante el verano a los Laboratorios de Psicología Experimental y a las oficinas de Orientación Profesional de Alemania y asistiendo al Congreso de Psicoanálisis que, presidido por Sigmund Freud, se celebró ese año en Berlín. Aunque al curso siguiente regresaría nuevamente al Instituto J.J. Rousseau, esta vez sólo por 3 meses, viaje igualmente subvencionado por la JAE.

\section{Desarrollo profesional}

Una de sus primeras actuaciones profesionales fue la preparación en 1923 - por encargo del Ministerio de Instrucción Pública- de un curso de cinco meses de duración sobre Técnicas Psicopedagógicas para maestros.

4 HERRERO, Fania. (2003): Mercedes Rodrigo: una pionera de psicología aplicada en España y Colombia. Tesis Doctoral. Madrid, Universidad Complutense, p. 119.

5 RODRIGO, Mercedes y ROSELLÓ, Pedro. (1922): "Lo que piensan de la guerra los niños españoles", en: Revista de Pedagogía 71, año 0. Publicado también en 1923 en L'Educateur, Febrero, pp. 422-425. 
Para ello elaboró con su compañero Pedro Roselló una herramienta que sería de utilidad fundamental entre las actividades psicotécnicas posteriores, el Registro Paidológico, ficha con la que entrenar a los maestros en la recogida de datos relativos a su alumnado que pudieran ser de utilidad para posteriores estudios psicopedagógicos de diversa aplicación.

Poco después, se crea el Instituto de Reeducación de Inválidos del Trabajo y Mercedes es nombrada encargada de la Sección de Orientación Profesional, terreno en el que podemos considerarla pionera y en el que trabajará a lo largo de toda su trayectoria profesional. De hecho diez años más tarde publicará una obra que recoge cuatro conferencias pronunciadas por ella, en las que muestra su interés recurrente por la orientación profesional ${ }^{6}$, reconociendo que éste había sido potenciado en sus tiempos de estudiante por su profesor E. Claparéde. Es de destacar en la primera de ellas su elogio al médico español Huarte de San Juan, a quien en la actualidad se le considera el patrón laico de la Psicología en España, por ser el precursor de la Psicología Diferencial.

Y ciertamente Juan Huarte de San Juan fue una figura pionera en toda Europa, pero es extraño que Mercedes, -siendo mujer- no denunciara explícitamente el sexismo de sus afirmaciones sobre la carencia de talento en las mujeres. O tal vez lo considerara natural por la mentalidad de la época en que fueron escritas. En cualquier caso es de suponer que, como cuando se hace eco de frases o ideas de Ramón y Cajal, de Rousseau o Freud, por poner algunos ejemplos, fija su atención tan sólo en lo positivo de la obra de los grandes maestros, pasando por alto sus misóginas afirmaciones ${ }^{7}$.

En 1923 publica con Roselló el trabajo por el que probablemente Mercedes Rodrigo ha sido más conocida, la traducción de los test de Claparéde ${ }^{8}$ en el que como novedad, rechazan la explicación genética y geográfica de las diferencias encontradas a favor de una explicación ambiental basada en la clase social. De hecho sus muestras, además de ser de un tamaño similar a las que utilizó su autor en Suiza, fueron obtenidas de niños y niñas madrileños pertenecientes a colegios tanto públicos como privados, encontrando en éstos últimos resultados mejores que en los de clases modestas, e incluso mejores que la media de los

6 RODRIGO, Mercedes. (1933): “Algunos problemas de Orientación profesional”. Medicina del Trabajo e Higiene Industrial, 17 y 18, pp. 93-160.

7 ... la razón de tener la primera mujer no tanto ingenio, le nació de haberla becho Dios fría y húmeda que es el temperamento necesario para ser fecunda y paridera y el que contradice el saber, y si la sacara templada como Adán, fuera sapientísima, pero no pudiera parir... (Huarte de San Juan, Examen de Ingenios para la Ciencia, 1575. Madrid, Edición de Felisa Fresco Otero, Espasa-Calpe).

8 RODRIGO, Mercedes, y ROSELLÓ, Pedro. (1923): "Revisión española de los tests de Claparéde (10ª serie)", en: Revista de Pedagogía, año II, No. 15, marzo 81-92. 
chicos suizos, hecho que atribuyeron a las pésimas condiciones socioculturales en que vivían los chicos/as de clases populares en Madrid.

Entre 1927 y 1936 trabajó con José Germain, que poco después sería el director del Instituto Nacional de Psicotecnia de Madrid, realizando tareas de Psicología experimental aplicada, fundamentalmente Psicometría para selección y orientación profesional. La creación en 1928 del Instituto de Psicotecnia implicó la puesta en marcha de una Red Nacional de Psicotecnia y la necesidad de impartir formación especializada al que habría de ser el personal psicotécnico, médico y administrativo de sus oficinas. En 1929 comenzaron a impartirse los primeros cursos en los que Mercedes Rodrigo fue docente destacada contribuyendo a organizar, estructurar y delimitar los diversos campos de aplicación, así como a entrenar y tutelar a quienes serían la segunda generación de profesionales de la Psicología en España.

En 1933 -fruto de su interés inicial por la superdotación- publicará con José Germain los primeros resultados de un test de inteligencia general ${ }^{9}$, sobre una muestra de 4.000 sujetos, después de una amplía revisión de los principales test de inteligencia al uso en todo el mundo. La prevención de accidentes, tema de absoluta actualidad en pleno siglo XXI, fue otra de las áreas en las que trabajó nuestra autora; prevención tanto en ámbitos laborales ${ }^{10}$ como de accidentes de tráfico, o prevención de accidentes infantiles ${ }^{11}$, insistiendo siempre en lo acertado y positivo de la prevención psicológica educativa (aunque sin negar los efectos de mostrar lo negativo de la falta de prevención).

...mientras sigan apareciendo diariamente en los periódicos sucesos reveladores de descuidos inconcebibles, atropellos frecuentísimos y estados de miseria que llevan a inocentes criaturas al último extremo de la desesperación seguiremos dando la voz de alarma y proponiendo remedios que, aunque no sean infalibles, tienden, por lo menos, a evitar gran número de desgracias ${ }^{12}$.

Cartel de prevención de accidentes de circulación de la Dirección General de Sanidad (Madrid) para los alumnos de escuelas primarias ${ }^{13}$.

9 GERMAIN, José y RODRIGO, Mercedes. (1933): "Primeros resultados de un test de inteligencia general". Archivos de Neurobiología, 4-6, pp. 1189- 1221.

${ }^{10}$ RODRIGO, Mercedes. (1929): La prevención de los accidentes de trabajo, en: La práctica médica en los accidentes de trabajo. OLLER, A. et al. Madrid, Morata.

11 RODRIGO, Mercedes. (1935): "Prevención de accidentes desde la infancia", en: Revista de Organización Científica, Vol. IV, No 29.

12 RODRIGO, Mercedes. (1935): Óp., cit, p. 686.

13 RODRIGO, Mercedes. (1935): Óp., cit, p. 686. 

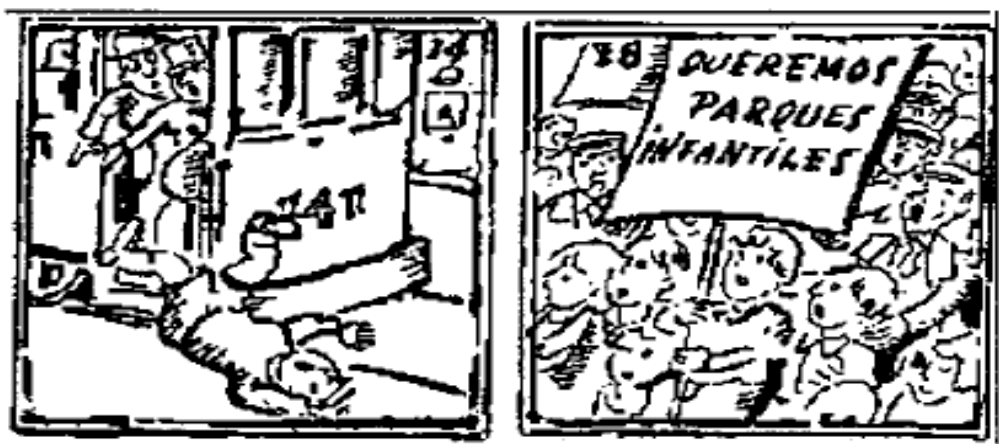

En 1936 fue nombrada directora del Instituto Nacional de Psicotecnia, algo que deja claramente en evidencia que fue bastante más que una asistente de Germain (como parece darse a entender en algunos escritos sobre su vida y su obra), cargo que ocupará hasta su exilio en 1939. La infancia problemática fue también objeto de su atención, razón por la que entre 1931 y 1939 trabajó como psicóloga clínica en el Tribunal Tutelar de Menores de Madrid y entre 1936 y 1937 en el Hogar de Delincuentes, llevando a la práctica -en su visión de la infancia desadaptada- las ideas psicopedagógicas de la Escuela Nueva.
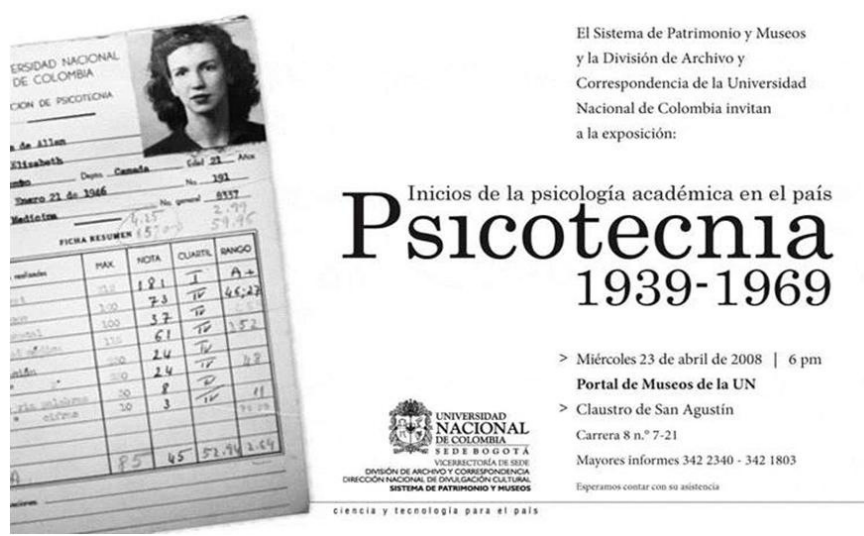

Su primera publicación al respecto es el artículo "Los niños malos" y la higiene mental"14, en donde pone de manifiesto su implicación con los principios educativos libertarios de Rousseau o con las ideas de Freud acerca de la etiología de las neurosis, que siempre tienen su origen en traumas infantiles.

Considera Mercedes que los chicos "malos" son fruto de la incomprensión o el desconocimiento de su entorno familiar y escolar, que parecen no tener otra misión que quitarles las ilusiones y contrariar sus tendencias naturales, que al verse truncadas reaccionan a veces perniciosamente. Y cuanto más les "pegan" sus padres, peor reaccionan ellos, además de ser un método inhumano, denigrante e ineficaz. E igual sucede con los maestros que aplican "el palo" a quienes no aprenden bien las lecciones. Para no tener que llegar a estos casos,

14 RODRIGO, Mercedes. (1934): "Los niños malos y la higiene mental", en:

Revista de Pedagogía. No. 151, pp. 310-318. 
tiene claro que más valdría prevenir que curar, incluso institucionalizando en escuelas hogares a quienes no tienen condiciones de vida dignas en sus familias, antes que recluirlos en "campos de concentración" para librar a la sociedad de sus malas acciones.

Poco antes de que terminara la guerra civil española nuestra autora -como muchas y muchos otros intelectuales y también pioneros de la Psicología, como por ej. Emilio Mira y López- abandona el país y parte al extranjero donde permanecerá hasta el final de sus días.

\section{Colombia: consolidación profesional}

Mercedes Rodrigo había sido invitada años atrás a Colombia por el entonces rector de la Universidad Nacional y fundador del Gimnasio Moderno- Agustín Nieto Caballero- para organizar los servicios de psicotecnia y seleccionar por primera vez al alumnado de nuevo ingreso a la Universidad. Y posiblemente ésta fue la razón que la impulsó a dirigir hacia allí sus pasosdespués de una breve estancia en Suiza- acompañada por su hermana María y por José María García Madrid, uno de sus principales colaboradores en el Instituto Nacional de Psicotecnia, llegando a Bogotá en Agosto de 1939.

En esa época, los pocos intelectuales españoles que se exilaron a Colombia fueron muy bien acogidos en un país ávido de su excelente cualificación, y esto fue lo que de hecho sucedió con ella, pues inmediatamente le facilitaron la creación de una Sección de Psicotecnia -con funciones de medición y evaluación psicológica-, asociada al Laboratorio de Fisiología de la Facultad de Medicina, al encontrar en el profesor Alfonso Esguerra Gómez, un incondicional admirador de la Psicología.

Antelos buenos resultados obtenidos en su primer curso por el alumnado que ella había seleccionado ${ }^{15}$, al poco tiempo se amplío la demanda de sus servicios extendiendo las labores de selección a otras Facultades y centros y también a instituciones y empresas no universitarias. Ya antes de iniciarse explícitamente un programa de formación en Psicología, comenzaron a impartirse cursos aislados, de actualización, para otros profesionales -sobre todo médicos-, pero sin un currículum completo ni estructurado.

${ }^{15}$ Ejemplo de ficha del Instituto de Psicotecnia, utilizada en 2008 para anunciar un evento. Es importante aclarar que en el buscador de imágenes de google aparece recortada la fotografía dando lugar a equívocos con la verdadera imagen de la propia Mercedes. Evidentemente no puede serlo porque además de que el nombre que figura es el de una chica llamada Elisabeth, de 21 años de edad, en la fecha que consta igualmente en la ficha -21 de Enero de 1946nuestra protagonista tendría 54 años al haber nacido el 13 de Mayo de 1891, según se refrenda en el Registro Civil del distrito de Buenavista en Madrid. 
Así por ejemplo en 1946, cuando se creó la cátedra de Psicología Médica en la Facultad de Medicina -a petición del profesor Esguerra Gómez-, Mercedes Rodrigo fue quien confeccionó el programa e impartió el primer año de docencia. Y de la recopilación de sus clases magistrales, surgiría una de las obras más completas para entender su visión de la Psicología: Introducción al Estudio de la Psicología (1949) ${ }^{16}$ cuyo índice reproducimos a continuación:

I. Psicología. Psicología y Medicina. Qué es la Psicología. Bosquejo Histórico. Divisiones de la Psicología. La Psicología en la actualidad.

II. Función Psicológica de la Infancia. Desarrollo del ser humano desde el nacimiento hasta los dos años.

III. Psicología del preescolar. Desarrollo de la conducta humana normal de $\operatorname{los} 3$ a los 7 años de edad.

IV. Psicología y educación. Años de escolaridad.

V. Tipos especiales de niños.

VI. "La declaración de los derechos del niño" y la delincuencia infantil.

VII. "Orientaciones sobre orientación profesional".

Finalmente el 20 de noviembre de 1947 -estando presente el Ministro de Educación Joaquín Estrada Monsalve y por supuesto la propia Mercedes Rodrigo que dirigió unas palabras- el Consejo Directivo de la Universidad Nacional con sede en Bogotá, emana el Acuerdo Número 231, cuyo contenido se encuentra en el Acta $n^{\circ} 44$ firmada por su rector, Gerardo Molina, que dice textualmente:

"Artículo $1^{\circ}$-Ampliar, a partir del $1^{\circ}$ de enero de 1948 la sección de Psicotecnia de la universidad, que a partir de esta fecha se denominará INSTITUTO DE PSICOLOGÍA APLICADA de la Universidad Nacional ${ }^{\prime \prime}$.

Realmente los primeros estudiantes no comenzarían sus clases hasta el 9 de Julio de 1948 en el nuevo Instituto compuesto a su vez por cinco secciones: Sección de Infancia y Adolescencia, Sección Universitaria, Sección de Investigación, Sección Psicomédica y Sección de Enseñanza (precursora esta última de lo que con los años se convertiría en la Facultad de Psicología, estando ya Mercedes en el exilio).

Además del ya mencionado Alfonso Esguerra, es justo reconocer que evidentemente hubo otras muchas personas que colaboraron con ella hasta poner en marcha la carrera de Psicología en Colombia. Especialmente

${ }^{16}$ RODRIGO, Mercedes. (1949): Introducción al estudio de la Psicología. Bogotá, Universidad Nacional.

17 Tomado de SILVA PERTUZ, Marta. (2007): La enseñanza de la Psicología en Colombia. El legado Rodriguista. Cambios y prospectivas. Psicogente, 10 (17): pp. 60-73. Universidad Simón Bolívar. Barranquilla, Colombia. 
la apoyó Luis López de Mesa (1884-1967), psiquiatra especializado en Harvard, que en 1920 elaboró el primer test psicológico colombiano, puso en marcha un programa de Cultura Aldeana... Ello además de ser rector de la Universidad Nacional, ministro de Educación y de Relaciones Exteriores, filósofo, sociólogo y escritor prolífico.

Por supuesto también estuvo siempre a su lado José María García Madrid, médico, psicólogo y colaborador activo desde 1939 en la Sección de Psicotecnia y desde 1948 en el

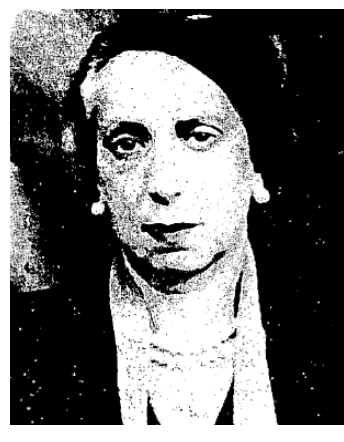
Instituto de Psicología Aplicada. Y otros muchos colaboradores como Julia Roncancio, Álvaro Villar Gaviria, Hernán Mendoza Hoyos, José Rodríguez Valderrama, Cecilia de Brigard y Alfonso Martínez Rueda.

A lo largo de sus 11 años de estancia en Colombia, la posición científica y personal de Mercedes se afianzó y amplió, participando activamente en encuentros y congresos internacionales, entre ellos el I Congreso Latinoamericano de Psicología, organizado en Montevideo en 1950 por Waclaw Radecki. También viajó a París, Ginebra, Washington y Moscú y realizó diversas publicaciones.

Del interés técnico que transmitían sus textos españoles, centrados en la adaptación y realización de pruebas objetivas de selección escolar o laboral; en su etapa colombiana mostró una mayor voluntad de servicio a la comunidad, preocupándose no sólo por el desarrollo y la divulgación de la ciencia, sino también implicándose éticamente con los profesionales de la Psicología en el desarrollo social del mundo de las posguerras, en una incansable actividad formativa e institucionalizadora.

Coincidiendo con Fania Herrero en las conclusiones de su análisis al minucioso trabajo de Mercedes Rodrigo sobre la profesión de Enfermería ${ }^{18}$, podemos con toda propiedad afirmar que "este tipo de trabajos no son sólo de psicología aplicada a un fin utilitario inmediato, o meros aunque novedosos estudios psicotécnicos, sino que, tomados en su conjunto, suponen una nueva labor fundacional de esta autora en Colombia: Las conclusiones de sus trabajos son casi estudios de psicología del pueblo colombiano, más o menos parciales según los casos, recuperando una tradición que se interesaba sobre las psicologías nacionales, ahora con el pleno aparato técnico que exige el método de una ciencia moderna"19.

${ }_{18}$ RODRIGO, Mercedes. (1944): Contribución al estudio psicológico de la profesión de enfermera. Universidad Nacional de Colombia, $\mathrm{N}^{\circ} 2$.

19 HERRERO, Fania. (2003): Óp., cit, p. 300. 
Pero al final -pese a todas sus aportaciones y a que llegó a sentir a Colombia como su segunda patria-, su condición de exiliada, su viaje a la Unión Soviética y su trabajo en la selección de aspirantes universitarios, la hicieron políticamente sospechosa y su vida y la de sus colaboradores comenzó a resultar insufrible. En palabras de Ardila ${ }^{20}$, la Colombia del 9 de abril de 1948 se parecía demasiado a la España de la Guerra Civil, por lo que -igual que entonces- tuvo nuevamente que abandonar el país que años atrás la había acogido con tanto entusiasmo.

Después de su partida, la Psicología en Colombia pasó por dos o tres décadas de auténtica confusión y desorientación. Aún en la actualidad, sólo un $20 \%$ de quienes estudian Psicología lo hacen en la Universidad Pública Nacional. En 1951 le cambiaron el nombre al Instituto de Psicología Aplicada para pasar a llamarse Instituto de Psicología, y en 1958 se aprobó finalmente el primer Plan de Estudios de la Facultad de Psicología, que ¿casualmente? tuvo una mujer por primera decana, la Licenciada Beatriz de la Vega.

El resto de la formación psicológica se oferta en universidades privadas -bien religiosas, bien laicas-, en 1962 se inician los estudios psicológicos en la Pontificia Universidad Javeriana, en 1968 en la Universidad de Los Andes y en 1970 en la Universidad Católica. También en esos años, el Instituto de Medicina Legal y Ciencias Forenses contrata a Victoria Eugenia Villegas Mejía, su primera psicóloga.

A partir de los años 70, se inauguran nuevas Facultades de Psicología a lo largo y ancho de Colombia hasta llegar al centenar aproximado actual, creándose a su vez la Asociación Colombiana de Facultades de Psicología, cuya primera presidenta fue nuevamente una mujer, Graciela Aldana de Conde.

\section{Puerto Rico y sus últimos años}

Mientras tanto, fueron otra vez -en éste el $2^{\circ}$ exilio de Mercedes Rodrigosu hermana María y José $\mathrm{M}^{\mathrm{a}}$ García Madrid quienes la acompañaron, viajando él primero a Puerto Rico y desde allí organizando el viaje para ellas en 1950. Una vez establecida, obtuvo trabajo como profesora de Educación en la Universidad de Puerto Rico, pues aún no existía la especialidad de Psicología.

También trabajó desde 1955 como consultora en la "Administración de veteranos" de EEUU, especializándose en la Clínica Juliá de San Juan en Psicóloga clínica. Allí permanecería hasta su jubilación, dedicándose a

${ }^{20}$ ARDILA, Rubén (comp.). (1993): Psicología en Colombia, Contexto Social e

Histórico. Bogotá, T.M. Editores. 
la Terapia tanto grupal como individual, actividad que al parecerle reportó grandes satisfacciones personales. En paralelo continúo como siempre con sus investigaciones psicométricas y con su interés por la Psicología aplicada, sin dejar nunca su activa vida profesional, publicando ${ }^{21}{ }_{-}^{22}$ y participando en congresos y reuniones científicas. De hecho, entre 1957-1959 fue vocal y luego presidenta de la Asociación de Psicología de Puerto Rico, de la que había sido socia fundadora en 1954.

A la muerte de su hermana María en 1968, Mercedes fue a vivir a casa de José $\mathrm{M}^{\mathrm{a}}$ García Madrid, que había contraído matrimonio unos años antes allí en Puerto Rico y que tenía ya tres hijos. En 1972, con 81 años, se jubiló definitivamente. Ciertamente ya estaba enferma, casi inválida y en consecuencia muy necesitada de ayuda, que le prestó gentilmente $\mathrm{M}^{\mathrm{a}}$ Teresa -la mujer de su siempre amigo y compañero-cuidándola con cariño hasta su muerte el 12 de Septiembre de 1982.

Afortunadamente al final de su vida -aunque no es su familia propiapudo al menos disfrutar del hogar que nunca tuvo, al no haberse casado ni tenido hijos por dedicar toda su vida al ejercicio profesional. Situación por otra parte bastante común en la mayoría de las pioneras de finales del XIX y principios del siglo XX en que, al no ser lo habitual que las mujeres trabajasen fuera del hogar, tenían serias dificultades para compaginar ambas dedicaciones.

\section{CONCLUSIÓN}

Mercedes Rodrigo Bellido vivió en Colombia entre los 49 y los 60 años -años sin duda idóneos para la consolidación profesional- y entre sus muchas actividades, con toda seguridad su mayor contribución fue la puesta en marcha del primer programa de formación en Psicología no sólo de Colombia, sino también de toda América Latina. Precisamente por ello el 20 de Noviembre ha sido declarado en Colombia Día del Psicólogo (aunque tendría que serlo también de la psicóloga, máxime cuando cuenta con una mujer pionera en sus orígenes, o de los y las profesionales de la Psicología, o simplemente de la Psicología), conmemorando con ello la fecha en que se creó la carrera en 1947.

Con los años se ha empezado a reconocerla importancia de Mercedes Rodrigo para la Historia de la Psicología tanto en España como en

${ }^{21}$ RODRIGO, Mercedes. (1954): "El problema de la deficiencia mental y sus derivaciones de tipo social y humanitario", en: Pedagogía (Universidad de Puerto Rico) 2 (2), pp. 107-158.

22 RODRIGO, Mercedes. (1955): Precursores españoles de la psicología moderna. Pedagogía Universidad de Puerto Rico 3 (2), pp. 137-116. 
Colombia, no sólo por sus méritos profesionales como psicóloga aplicada pionera, sino también por haber servido de puente entre la psicopedagogía española y la europea: divulgando incansablemente las teorías y principios de la Psicología moderna y de la Psicopedagogía, participando activamente en la organización y el funcionamiento de diversas instituciones pedagógicas y científicas, y manteniendo vivas las relaciones entre los y las investigadoras pensionadas de la Junta de Ampliación de Estudios y las y los maestros de Ginebra -especialmente Claparéde y Piaget- a través de la Asociación Española de antiguos alumnos y Amigos del Instituto J.J. Rousseau.

Así, testmonia Ardila ${ }^{23}$ que Mercedes Rodrigo regresaría a Colombia en Octubre de 1971 para recibir el Primer Premio Nacional de la Federación Colombiana de Psicología, correspondiente al año anterior:

"por su gran labor pionera a favor del desarrollo de la Psicología cientifica y profesional en Colombia primero, desde la Sección de Psicotecnia, adscrita a la Facultad de Medicina de la Universidad Nacional de Colombia, y luego desde el Instituto de Psicología Aplicada de la misma Universidad, estructuró la psicología colombiana, le dio su forma actual, y entrenó a quienes iban a convertirse en los líderes de la psicología de las siguientes décadas. Su fe en el papel central que tiene la Psicología aplicada en el desarrollo socioeconómico de los pueblos, su celo por la solidez metodológica de las investigaciones, su respeto por las implicaciones éticas de la Psicología cientifica y profesional, se han perpetuado en varias generaciones de psicólogos. Nuestra asociación quiere destacar en esta forma la labor cumplida por Mercedes Rodrigo Bellido, principal figura de la Historia de la Psicología colombiana y punto de iniciación de la Psicología en nuestro país ${ }^{24}$.

E igualmente, refiriéndose a España, Fania Herrero ${ }^{25}$ comenta: "por todas estas razones su papel en la creación y desarrollo de nuestra Psicología Aplicada, original, creativo, extraordinariamente activo, está fuera de toda duda"... lo que contrasta con "la escasa atención que esta autora había venido atrayendo a lo largo de sus exilios, creemos que como un acto de injusticia intencionado por parte, especialmente, de José Mallart, quien tuvo y ejerció el poder de delimitar el espectro de la Psicología merecedora de consideración" por su papel de redactor de la Revista de Psicología General y Aplicada.

23 ARDILA, Rubén. (1973): La Psicología en Colombia. Desarrollo Histórico.

México, Trillas, pp. 32 y 33.

${ }^{24}$ Ibídem., p. 32 y 33.

25 HERRERO, Fania. (2003): Óp., cit, p. 393. 
Por nuestra parte no pensamos que su olvido durante décadas se haya debido a un solo autor, más bien parece fruto de los tiempos en que las mujeres eran la excepción y por eso su presencia apenas pasaba de la mera anécdota. Afortunadamente en la actualidad, los estudios sobre las mujeres están empezando a rescatar a muchas pioneras que, como ella, contribuyeron a poner las bases de nuestro actual conocimiento científico.

Como relata Ardila (1988), antes de ella en Colombia la Psicología era una disciplina que abordaban especialmente los médicos en sus tesis de grado, o los teólogos en sus elucubraciones filosóficas. Pero los educadores, pese a saber de su importancia, no sabían ni pretendían utilizarla. Ella, por el contrario insistió en que la Psicología tenía su propio campo de trabajo, distinto aunque aplicable tanto en Medicina, como en Filosofía y por supuesto en Educación.

\section{FUENTES}

RODRIGO, Mercedes. (1925): Breves consideraciones sobre la ficha informativa. Memorias del Instituto de Reeducación de Inválidos del Trabajo, No. 2. Madrid.

RODRIGO, Mercedes. (1925): Protección de los niños contra los accidentes. Memorias del Instituto de Reeducación de Inválidos del Trabajo. No. 2. Madrid.

RODRIGO, Mercedes. (1926): Congreso Internacional de Orientación Profesional Femenina. Memorias del Instituto de Reeducación de Inválidos del Trabajo, 3. Madrid.

RODRIGO, Mercedes. (1927). III Congreso Internacional de Organización Cientifica del Trabajo. Memorias del Instituto de Reeducación de Inválidos del Trabajo, No. 4.

RODRIGO, Mercedes. (1927): La orientación Profesional femenina. Recopilación de los Trabajos del IV Congreso de Estudios Vascos.

RODRIGO, Mercedes. (1928): "Trabajo de totalización de las respuestas recibidas a las preguntas sobre consultas públicas gratuitas que la Junta de Gobierno del Colegio de Madrid dirigió a sus colegiados", en: Boletín del Colegio de Médicos de la Provincia de Madrid, año X, No. 121, Nov. 235-253. Madrid.

RODRIGO, Mercedes. (1929): La prevención de los accidentes del trabajo, en: La práctica médica en los accidentes de trabajo. OLLER, A. et al, Madrid, Morata. 
RODRIGO, Mercedes. (1930): La Psicotecnia en la prevención de los accidentes de trabajo. Medicina del Trabajo e Higiene Industrial, 6.

RODRIGO, Mercedes. (1931): "Métodos psicopedagógicos para la prevención de accidentes". Conferencia en el Instituto Universitario de Ciencias de la Educación de Ginebra. Madrid, Organización Científica, Vol. 2, No.14.

RODRIGO, Mercedes. (1932):Para prevenir accidentes del trabajo. Vida de negocios. Madrid.

RODRIGO, Mercedes. (1933):Algunos problemas de Orientación profesional. Medicina del Trabajo e Higiene Industrial, 17 y 18.

RODRIGO, Mercedes. (1933): Aspectos psicopedagógicos de la selección del superdotado. Madrid, Artes Gráficas Municipales.

RODRIGO, Mercedes. (1934): "Los niños malos y la higiene mental", en: Revista de Pedagogía No. 151.

RODRIGO, Mercedes. (1934): “Cómo utilizar para la orientación profesional el último año de escolaridad", comunicación presentada al Congreso Internacional de Enseñanza Técnica, de Barcelona", en: Revista de Organización Científica, Vol. IV, No 25.

RODRIGO, Mercedes. (1935): "Prevención de accidentes desde la infancia", en Revista de Organización Científica, Vol. IV, No. 29.

RODRIGO, Mercedes. (1941): Conozca al Niño. Educación, No 2. Bogotá.

RODRIGO, M. (1941): El niño y su medio. Educación, No 2. Bogotá.

RODRIGO, Mercedes. (1942): El problema de los accidentes y la acción social de la escuela. Alma Nacional, Vol. 1.

RODRIGO, Mercedes. (1942): "Niños españoles en tiempos de guerra”, en: Revista Española. Bogotá.

RODRIGO, Mercedes. (1942): Informe de la sección de Psicotecnia. Bogotá, Minerva.

RODRIGO, Mercedes. (1942):"Encuesta sobre los motivos que impulsan a ingresar en la Universidad”, en: Revista Facultad de Medicina, No.4.

RODRIGO, Mercedes. (1944): Contribución al estudio psicológico de la profesión de enfermera. Universidad Nacional de Colombia, $\mathrm{N}^{\mathrm{o}} 2$.

RODRIGO, Mercedes. (1946): Quépuede hacer la Psicotecnia por el estudiante. Universidad Nacional de Colombia, $\mathrm{N}^{\circ} 6$.

RODRIGO, Mercedes. (1949): Instituto de Psicología aplicada: Fundación y Plan de Estudios. Bogotá, Universidad Nacional.

RODRIGO, Mercedes. (1949): Introducción al estudio de la Psicología. Bogotá, Universidad Nacional. 
RODRIGO, Mercedes. (1955): Precursores españoles de la Psicología moderna. Pedagogía. Universidad de Puerto Rico.

RODRIGO, Mercedes y GARCÍA MADRID, José Ma . (1944): Contribución al estudio psicológico de la profesión de enfermera. Universidad. Nacional de Colombia, No 1.

RODRIGO, Mercedes y ROSELLÓ, Pedro. (1922): "Lo que piensan de la guerra los niños españoles", en: Revista de Pedagogía No. 71, año 0, 422425. Publicado también en 1923 en L'Educateur, Febrero.

RODRIGO, Mercedes, y ROSELLÓ, Pedro. (1923): "Revisión española de los tests de Claparéde ( $10^{a}$ serie)", en: Revista de Pedagogía, año II, No 15 , marzo.

\section{REFERENCIAS}

ARDILA, Rubén. (comp.) (1993): Psicología en Colombia, Contexto Social e Histórico. Bogotá, T.M. Editores.

ARDILA, Rubén. (1988): Mercedes Rodrigo (1891-1982), en: Revista Latinoamericana de Psicología, Vol. 20 No. 3.

ARDILA, Rubén. (ed.) (1986): La Psicología en Américo Latina: Pasado, Presente y Futuro. México, Siglo XXI.

ARDILA, Rubén. (1973): La Psicología en Colombia. Desarrollo Histórico. México, Trillas.

ARDILA, Rubén. (1970): "Applied Psychology in Colombia”, en: Rev. of App. Psych, No. 19.

ARDILA, Rubén. (1969):“Desarrollo de la Psicología Latinoamericana.”, en: Revista Latinoamericana de Psicología Vol.1, No. 1.

BOIX, Montserrat (2005). "La historia de las mujeres, todavía una asignatura pendiente". Extraído desde internet (online): mujeresenred.net. http:// www.mujeresenred.net/spip.php?article272\#nb11

CARPINTERO, Helio; HERRERO, F., y PARAJÓN, L. (1995): "Los emigrados de la Psicología Española y su proyección Iberoamericana", en: VVAA: Un siglo de Psiquiatría en España. Madrid, Extra Editorial

GARCÍA COLMENARES, Carmen. Autoridad femenina y reconstrucción biográfica: el caso de las primeras psicólogas españolas.http:/ /webs.uvigo. es/reined/ojs/index.php/reined/article/viewFile/22/13 
GARCÍA DAUDER, S. (2005): Psicología y feminismo. Historia olvidada de mujeres pioneras en Psicología. Madrid, Narcea.

HERRERO, Fania, GARCÍA, E., y CARPINTERO, Helio. (1995): "Psicopedagogía en España (1900-1936). Becarios españoles en centros europeos", en: Rev. Hist. Psicol No. 16 (1-2).

HERRERO GONZÁLEZ, Fania. (2003): Mercedes Rodrigo: una pionera de psicología aplicada en España y Colombia. Tesis Doctoral leída el 17 de Mayo de 2000 en la Facultad de Filosofía de la Universidad Complutense de Madrid. Dirigida por Heliodoro Carpintero y Emilio García. http:/ / eprints. ucm.es/tesis/19972000/H/2/H2095901.pdf

JARAMILLO, J. (1989): La educación durante los gobiernos liberales. 1930 1946. Nueva Historia de Colombia. Tomo IV. Bogotá, Planeta.

MARIN, M.T. (1990): La renovación pedagógica en España (1907-1936). Los pensionados en pedagogía por la Junta de ampliación de estudios. Madrid, Consejo Superior de Investigaciones Científicas.

MARIN, M.T. (1991): Innovadores de la educación en España. Servicio de Publicaciones de la Universidad de Castilla La Mancha.

MARIN, M.T. (2002): Mujeres en la europeización de España (1907- 1936), en Las mujeres en la construcción del mundo contemporáneo. MARÍN, T. y POZO, M. M. del (eds.). Cuenca, Diputación de Cuenca.

MARÍN, T. (1990): La renovación pedagógica en España (1907-1936). Los pensionados en pedagogía por la Junta para ampliación de estudios. Madrid, CSIC.

MARTÍNEZ GORROÑO, Ma Eugenia. (1999): Españolas en Colombia. La buella cultural de mujeres exiladas tras la guerra civil. Madrid, Fundación "Españoles en el Mundo".

MARTÍNEZ CARROÑO, Ma Eugenia (1990):"Fuentes orales para una aproximación al exilio femenino en Colombia. Espacio, Tiempo y Forma”, en: Revista de la Facultad de Geografía e Historia, UNED Madrid.

MARTÍNEZ GUTIÉRREZ, J. (2002): Las intelectuales. De la segunda república al exilio. Alcalá de Henares, Ayuntamiento Alcalá de Henares. Concejalía de la Mujer.

MINISTERIO DE INSTRUCCIÓN PÚBLICA. (2003): Expediente académico de Mercedes Rodrigo en la Escuela Normal de Maestras de Madrid. AGA, Educación.

MOLERO, A. y POZO, M.M. (1989): La Escuela de Estudios Superiores del Magisterio (1909-1932). Alcalá de Henares, Universidad de Alcalá. 
Programa para la obtención del título de Psicólogo. Acuerdo del consejo directivo de la Universidad Nacional de Colombia n ${ }^{\circ} 13,3$ de marzo de 1958. Revista de Psicología, Bogotá, Vol. III, No 1-2.

UNIVERSIDAD NACIONAL DE COLOMBIA. (1949): Solicitud de expedición de pasaporte para Mercedes Rodrigo. AGA, Asuntos Exteriores, 3131.

GERMAIN, José, y RODRIGO, Mercedes. (1930): Pruebas de Inteligencia. Madrid, La Lectura.

GERMAIN, José; y RODRIGO, Mercedes. (1933):“Primeros resultados de un test de inteligencia general", en: Archivos de Neurobiología.

GERMAIN, José; RODRIGO, Mercedes, y MALLART, José. (1933). "La Psicofisiología en la organización del tráfico", en: Boletín de la Institución Libre de Enseñanza.

GONZÁLEZ, Ana (coord.) y LOMAS, Carlos (coord.) (2002): Mujer y educación: educar para la igualdad, educar desde la diferencia". Eds. Graó.

PEÑA, T.E. (1986): La Psicología en Colombia: Historia de una disciplina y una profesión. Bogotá, Ciencia, Tecnología y Desarrollo.

ROCA DE TORRES, Y. (1993): "La A.P.P.R.: Una perspectiva histórica”, en: Revista Puertorriqueña de Psicología, Vol. 9.

ROCANCIO, (1956): "El Instituto de Psicología en la Universidad Nacional”, en: Revista de Psicología No. 1. Bogotá.

RODRIGUEZ, W. (1993): Orígenes, en: Psicología en Colombia. Contexto Social e Histórico. ARDILA, Rubén. Bogotá, T.M. editores.

ROSA, A.; QUINTANA, y LAFUENTE, Enrique (eds.) (1988): Psicología e Historia. Contribuciones a la investigación en Historia de la Psicología. Madrid, Eds. UAM.

SÁEZ DE LA CALZADA, C. (1976): "Educación y pedagogía", en El exilio español de 1939. Tomo III Revistas, Pensamiento y Educación. ABELLÁN, J. L. y otros (dirs).Madrid, Taurus.

SÁIZ, M. y SÁIZ, B. (1998): "La Psicología aplicada en España”, en: Hin. Psicol, Vol. 19 (1).

SÁNCHEZ RON, J.M. (coor.) (1988): La Junta para Ampliación de Estudios e Investigaciones científicas, ochenta años después (2 vols.). Madrid, CSIC.

SILVA PERTUZ, Marta. (2007): La enseñanza de la Psicología en Colombia. El legado Rodriguista. Cambios y prospectivas. Psicogente. Barranquilla, Colombia. Universidad Simón Bolívar. 
ZABALA, Iris. (1991): "El exilio español y la marginación cultural", en: Cincuenta años de exilio español en Puerto Rico y el Caribe 1939-1989. CORUÑA, A, Ediciós do Castro.

GUIL BOZAL, Ana y VERA GIL, Sara. (2011): "Entre Europa y América Latina: Mercedes Rodrigo, psicopedagoga pionera" en: Revista Historia de la Educación Latinoamericana Vol. 13 No. 17, Tunja, Universidad Pedagógica y Tecnológica de Colombia, RUDECOLOMBIA, SHELA- HISULA pp. 71-92 